\title{
Supporting Information: Range, Magnitude and Ultrafast Dynamics of Electric Fields at the Hydrated DNA Surface
}

Torsten Siebert, Biswajit Guchhait, Yingliang Liu, Benjamin Fingerhut, Thomas Elsaesser

Max-Born-Institut für Nichtlineare Optik und Kurzzeitspektroskopie, D-12489 Berlin, Germany

\section{Preparation of DNA samples}

Salmon testes DNA water films with sodium counterions were prepared with an aqueous 0.1 $\mathrm{M} \mathrm{NaCl}$ solution. The $\mathrm{H}_{2} \mathrm{O}$ to DNA ratio was $\mathrm{N} \geq 150$ water molecules per base pair. In a second sample, the $\mathrm{Na}^{+}$counterions were replaced by $\mathrm{Mg}^{2+}$ with the corresponding $1.0 \mathrm{M}$ $\mathrm{MgCl}_{2}$ solution. As a reference, we studied $\sim 10 \mu \mathrm{m}$ thick films of salmon testes DNA with cetyl-trimethyl-ammonium $\left(\mathrm{CTMA}^{+}\right)$counterions, ${ }^{1}$ held in a humidity cell to maintain a welldefined hydration level. ${ }^{2}$ At 0 and $92 \%$ relative humidity (r.h.), the sample films contain $\mathrm{N}<2$ and $\mathrm{N} \approx 20-30$ water molecules per base pair. The latter hydration level corresponds to a statistical distribution of water molecules within the first two layers of the hydration shell.

\section{Two-dimensional (2D) infrared spectra}

\subsection{Experimental method}

The 2D infrared spectra were derived from 3-pulse photon echo experiments. ${ }^{3-5}$ The nonlinear signal emitted in the direction $\mathrm{k}_{\mathrm{s}}=-\mathrm{k}_{1}+\mathrm{k}_{2}+\mathrm{k}_{3}\left(\mathrm{k}_{\mathrm{i}}\right.$ : wavevector of pulse $\left.\mathrm{i}\right)$ was heterodyned with a local oscillator pulse and detected in a frequency resolved way (resolution $2 \mathrm{~cm}^{-1}$ ). Infrared pulses with a duration of $125 \mathrm{fs}$ were generated by parametric frequency conversion from an amplified Ti:sapphire laser system (repetition rate $1 \mathrm{kHz}$ ). ${ }^{6}$ The spectral bandwidth of the mid-infrared pulses was $160 \mathrm{~cm}^{-1}$ FWHM centered at $1030 \mathrm{~cm}^{-1}$ and their energy on the DNA sample approximately $1 \mu \mathrm{J} /$ pulse. All $2 \mathrm{D}$ spectra were recorded with parallel linear polarization of the 3 pulses interacting with the DNA sample.

\subsection{Experimental spectra}

The 2D spectra presented in Fig. 2A-C of the article were recorded at a waiting time of $\mathrm{T}=500$ fs. To assess potential changes of the $2 \mathrm{D}$ lineshapes and cross-peak patterns, series of $2 \mathrm{D}$ spectra were generated up to the maximum waiting $\mathrm{T}$ at which measurements with a sufficient signal to noise ratio are possible. Figure S1 A and C display 2D spectra of the DNA water film for waiting times of $\mathrm{T}=1.0$ and $1.5 \mathrm{ps}$. 


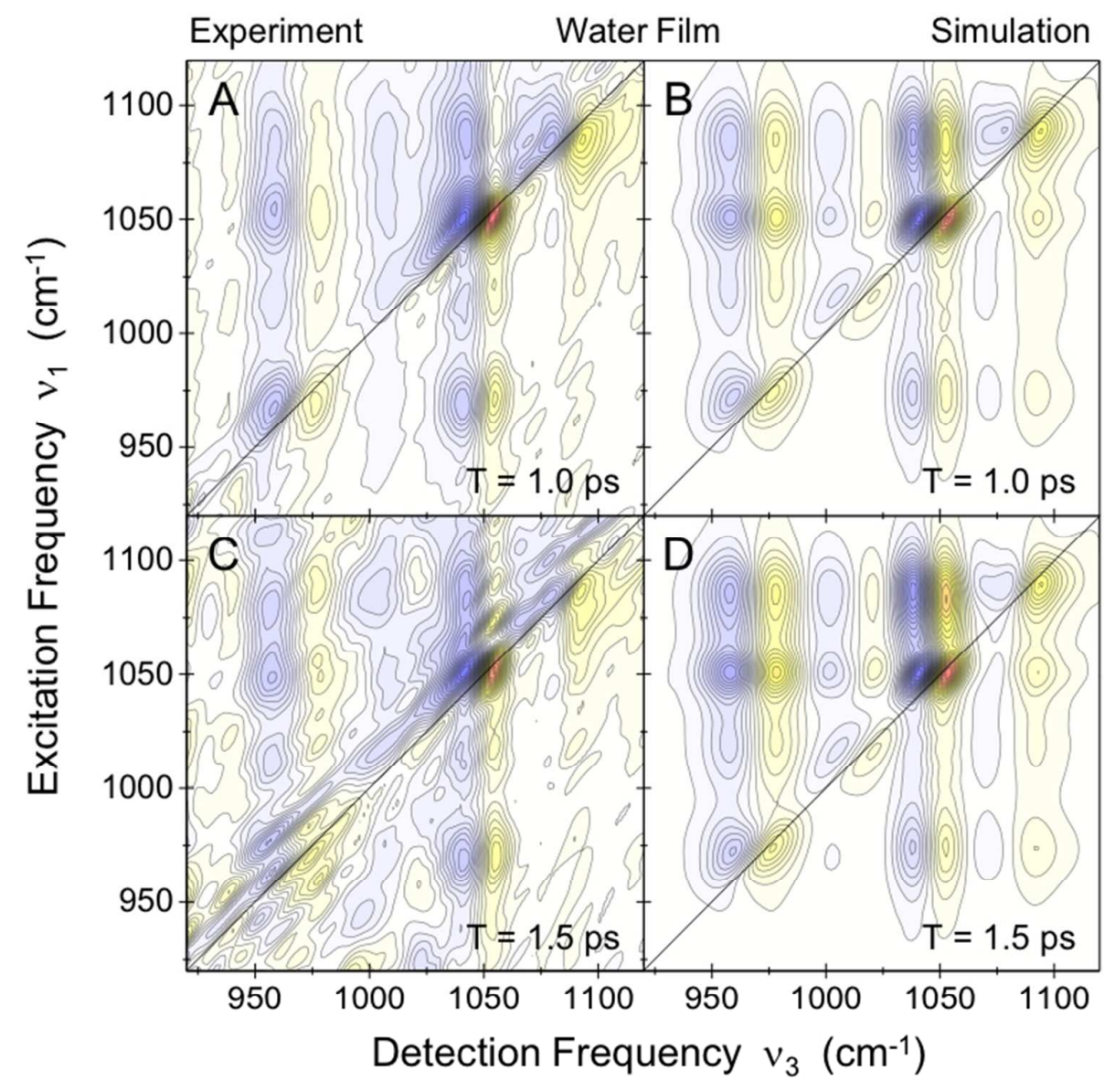

Figure S1. Experimental $(A, C)$ and calculated (B,D) 2D infrared spectra of the backbone modes at extended waiting times for water films of salmon DNA with $\mathrm{N}>150$ water molecules per base pair. The parameters derived from the simulation are given in Table S1.

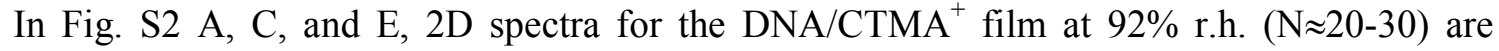
shown for waiting times up to $\mathrm{T}=4$ ps while spectra of the dehydrated film $(0 \%$ r.h., $\mathrm{N}<2)$ are presented for the same waiting times in Fig. S3 A, C, and E. In all cases, changes of the 2D lineshapes with increasing $\mathrm{T}$ are minor. The amplitudes of diagonal and cross peaks from intermode coupling change with increasing waiting times due to vibrational relaxation and down-hill population transfer between the backbone modes. 


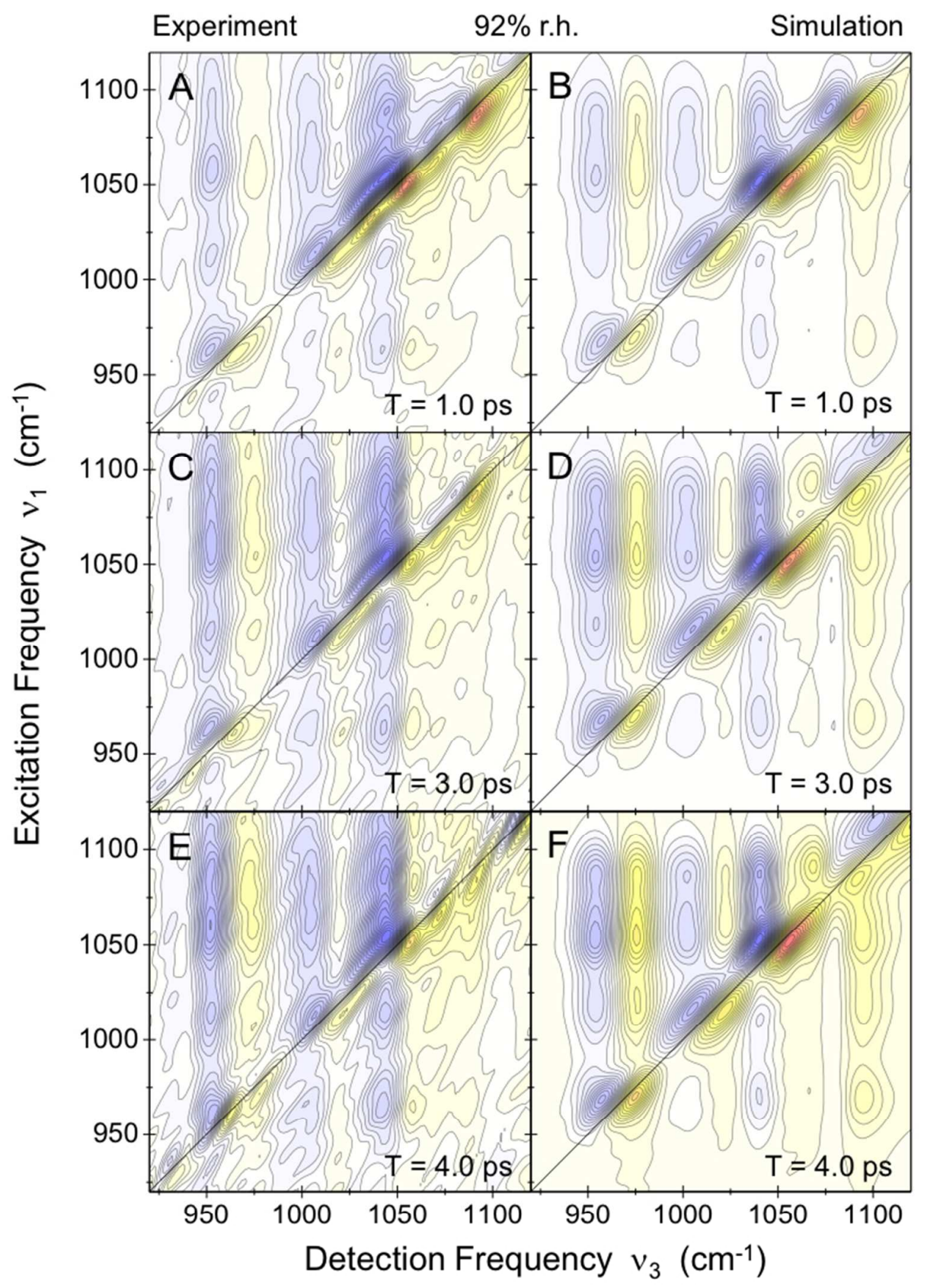

Figure S2. Experimental (A,C,E) and calculated (B,D,F) 2D infrared spectra of the backbone modes at extended waiting times for $\mathrm{DNA} \mathrm{CTMA}^{+}$films at $92 \%$ r.h. ( $\approx \approx 20-30$ water molecules per base pair). The parameters derived from the simulation are given in Table S2. 


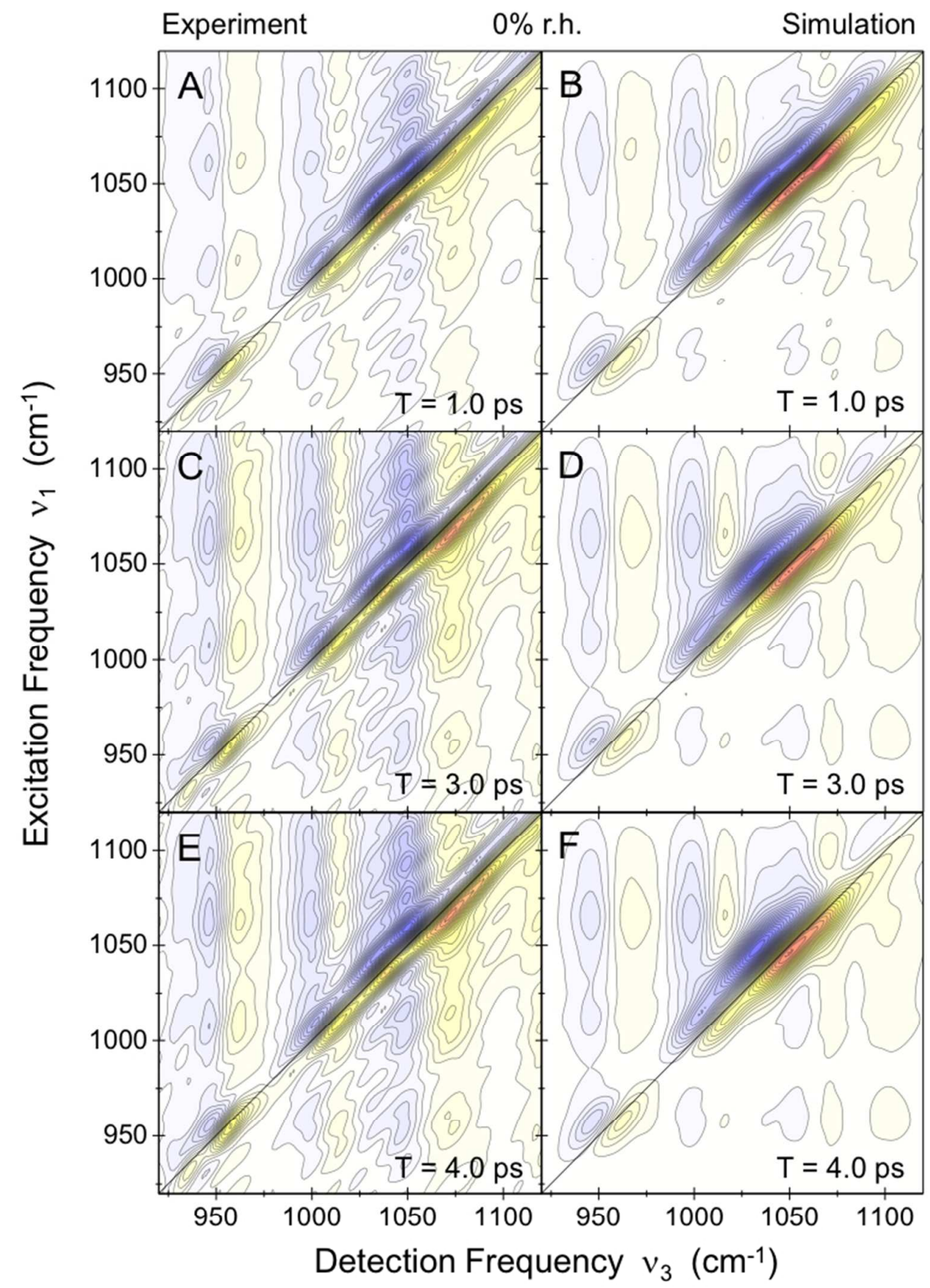

Figure S3. Experimental (A,C,E) and calculated (B,D,F) 2D infrared spectra of the backbone modes at extended waiting times of the DNA/CTMA ${ }^{+}$films at $0 \%$ r.h. $(\mathrm{N}<2$ water molecules per base pair). The parameters derived from the simulation are given in Table S3.

An exchange of counterions has a negligible effect on the 2D lineshapes. This is evident from the 2D spectra taken with $\mathrm{Na}^{+}$and $\mathrm{Mg}^{2+}$ in the DNA water film (Fig. S4), again illustrating the minor role of counterions as a source of fluctuating electric fields at the DNA/water interface. 


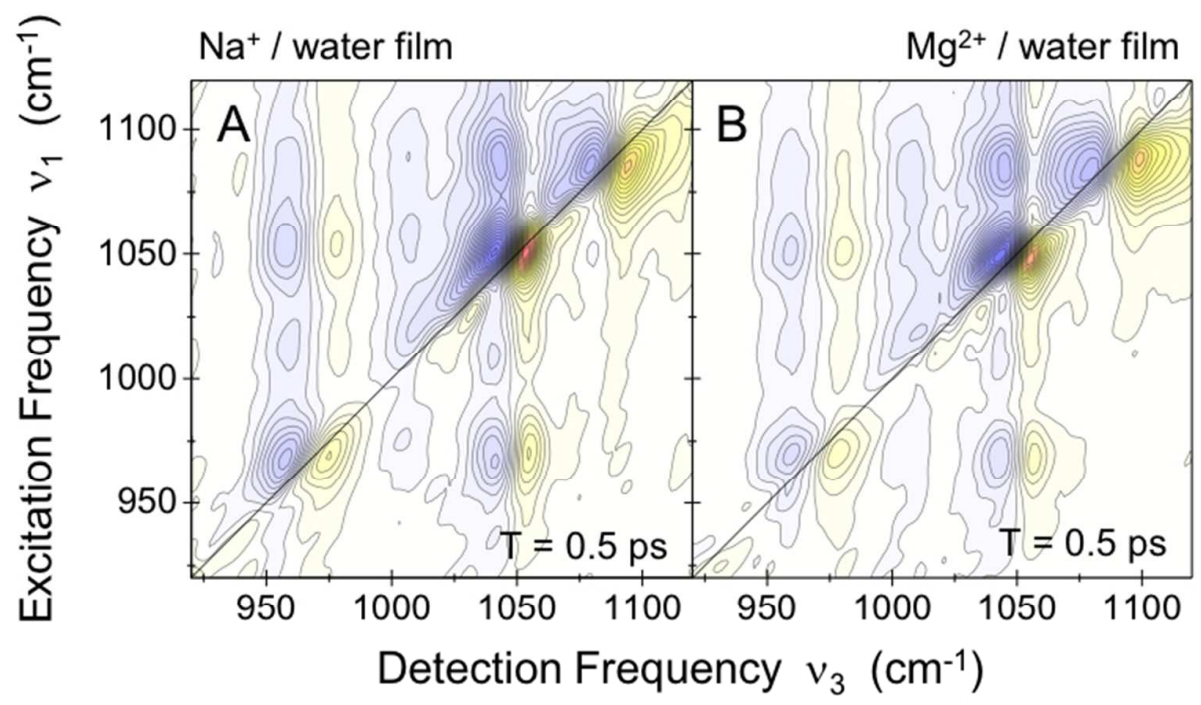

Figure S4. 2D infrared spectra of DNA water films prepared with (A) an $0.1 \mathrm{M} \mathrm{NaCl}$ and (B) a $1.0 \mathrm{M} \mathrm{MgCl}_{2}$ (B) buffer solution. The exchange of counterions has a negligible influence on the $2 \mathrm{D}$ spectra.

\subsection{Analysis of 2D spectra}

The experimental 2D spectra are analyzed by calculating the third-order response functions to the photon-echo pulse sequence in an approach based on perturbation theory of light-matter interaction, Kubo lineshape analysis, and a rate equation system to characterize vibrational population transfer between the backbone modes. A detailed description of this treatment has been given in the extended supplement of Ref. 7. Simulations were performed for the full set of $2 \mathrm{D}$ spectra, i.e., for all hydration levels and waiting times $\mathrm{T}$, and incorporate the full set of diagonal and cross peaks in order to address their pronounced spectral overlap and partial compensation. This in turn calls for a full model of the dynamics of vibrational relaxation and energy exchange between backbone modes. The interaction with the fluctuating aqueous environment is included via the lineshape parameters and the underlying frequency fluctuation correlation function (FFCF). Changes in the fluctuating electric fields and/or local hydrogen bond geometries at different hydration levels are reflected in changes of lineshape parameters, primarily in the signals located along the frequency diagonal. As an independent benchmark, the linear infrared absorption spectra at the different hydration levels are calculated, using the parameters extracted from the analysis of the 2D spectra.

The frequency fluctuation correlation function is given by the Kubo ansatz,

$$
\left\langle\delta v_{i}(t) \delta v_{i}(0)\right\rangle=\Delta_{1, i}^{2} \exp \left(-t / \tau_{1}\right)+\Delta_{2, i}^{2} \exp \left(-t / \tau_{2}\right)
$$

where $\delta v_{\mathrm{l}}(t)$ represents the frequency excursion of mode $i$ at time $t$ from its average transition frequency, $\Delta_{1, i}$ and $\Delta_{2, i}$ are fluctuation amplitudes, and $\tau_{1}$ and $\tau_{2}$ the related correlation times. 
The lineshapes of all diagonal peaks at the different hydration levels were reproduced by keeping the values of $\tau_{1}=300$ fs and $\tau_{2}>10$ ps constant, yielding mode-specific values for the fluctuation amplitudes.

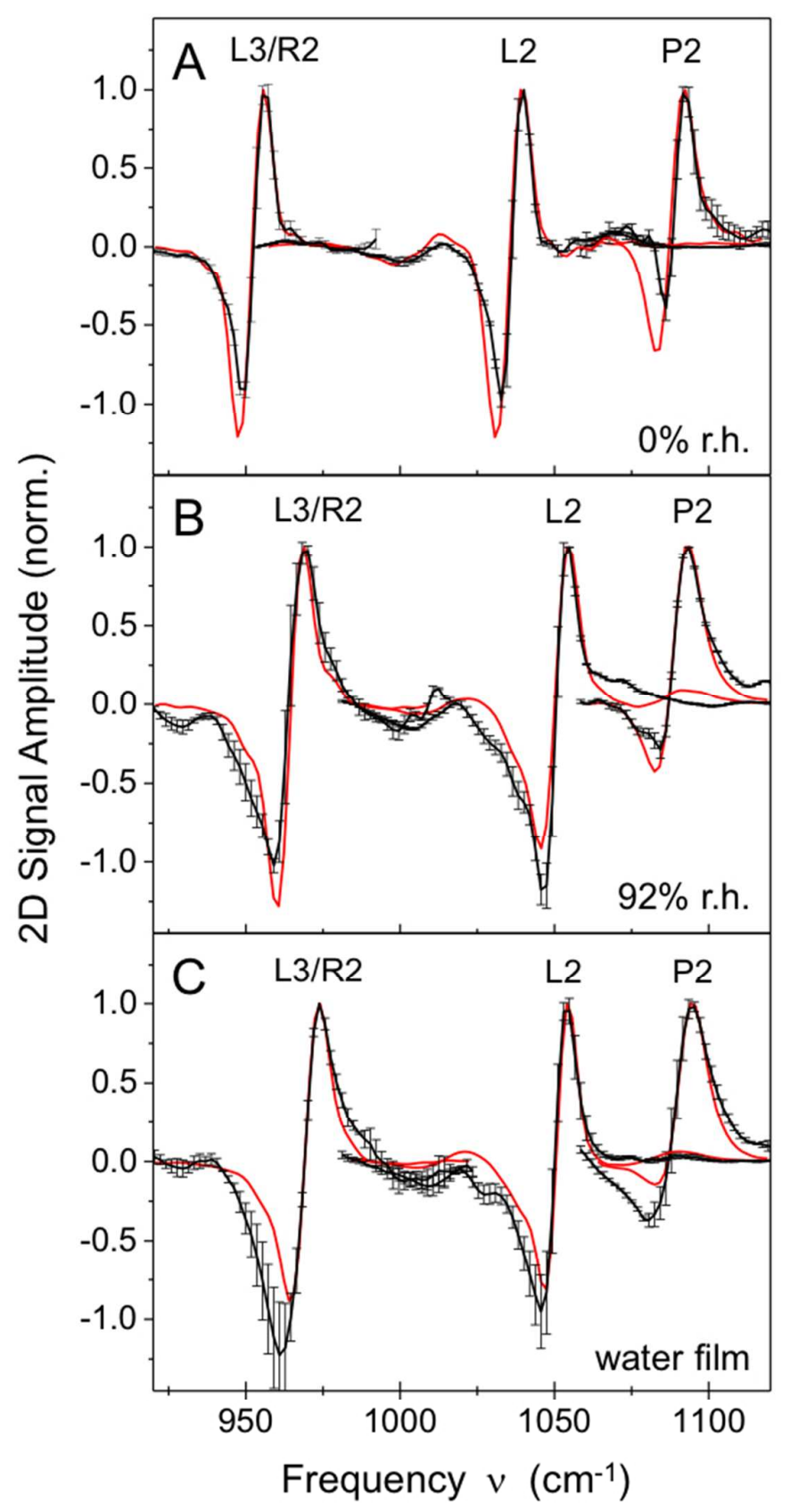

Figure S5. Comparison of the antidiagonal profiles of selected backbone resonances in the calculated 2D spectra at $\mathrm{T}=500 \mathrm{fs}$ (red lines, cf. Fig. 2 of the article) and anti-diagonal cuts of the experimental 2D spectra presented in Fig. 2 and 3 of the article (black lines). 
In Fig. 2D-F of the article and Figs. S1-S3, the calculated 2D spectra are plotted in direct comparison with the experimental spectra, Fig. S5 shows a comparison of calculated and experimental profiles along antidiagonals in the $2 \mathrm{D}$ frequency plane. The calculations reproduce the key features of the measured 2D spectra in a quantitative way, with minor deviations in the anti-diagonal profiles.

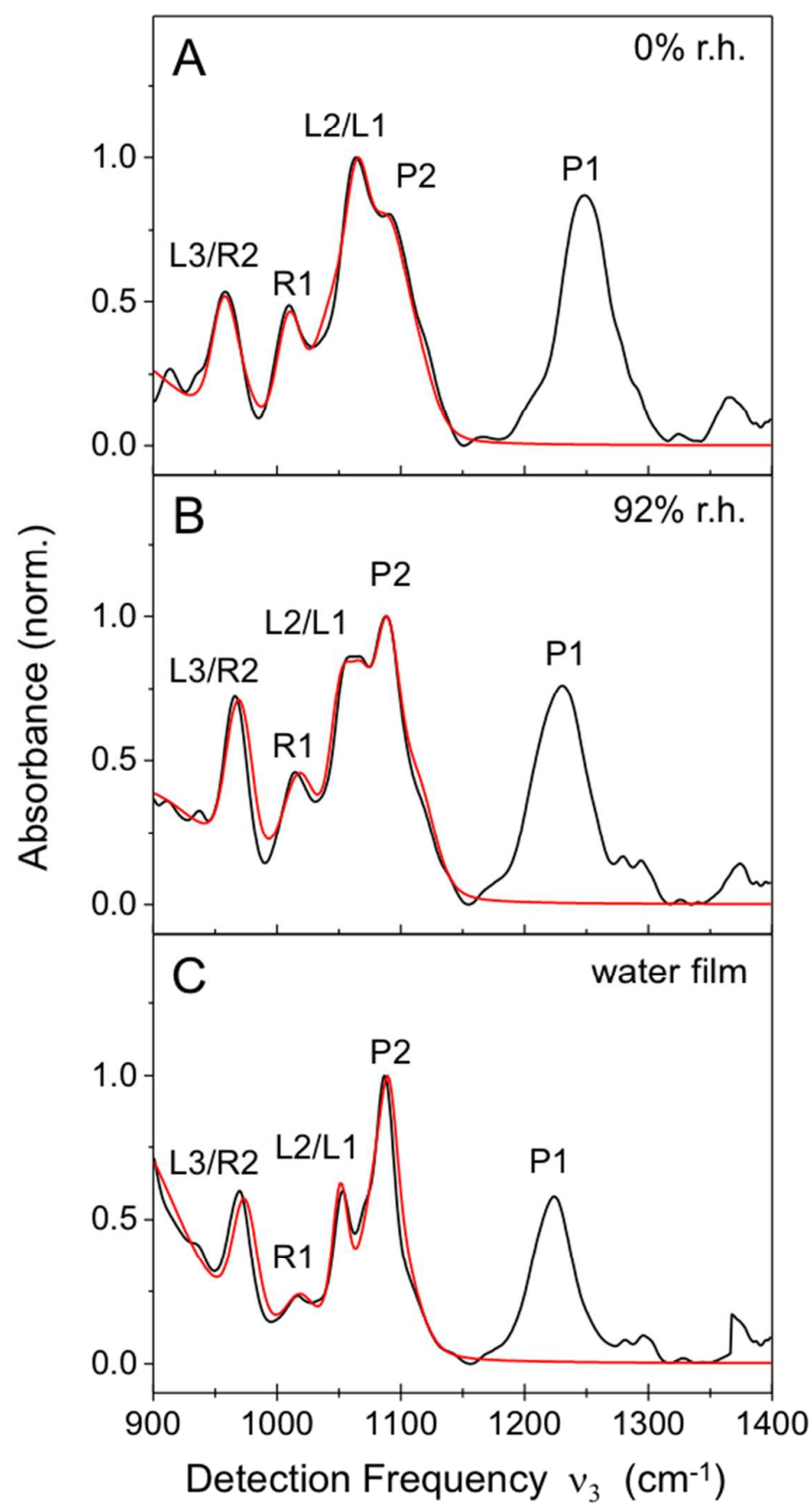

Figure S6. Experimental and calculated linear infrared absorption spectra of salmon DNA at (A) $0 \%$ r.h., (B) $92 \%$ r.h., and (C) in water films of a $0.1 \mathrm{M} \mathrm{NaCl}$ buffer. The parameters used for calculating the stationary spectra are taken from the simulation of the $2 \mathrm{D}$ spectra. 
The extracted line shape parameters are summarized in Tables S1-S3, including the (global) time constants and rates for down-hill $\left(\tau_{\mathrm{d}}, \mathrm{k}_{\mathrm{d}}\right)$ and up-hill $\left(\tau_{\mathrm{d}}, \mathrm{k}_{\mathrm{d}}\right)$ energy transfer between the modes. Experimental and calculated linear infrared spectra are compared in Fig. S6.

Table S1: Parameters of the backbone modes for salmon DNA in a water film of $0.1 \mathrm{M} \mathrm{NaCl}$.

\begin{tabular}{|c|c|c|c|c|c|c|}
\hline Mode & $\begin{array}{c}\text { Frequency } \\
\boldsymbol{v} / \mathbf{c m}^{-\mathbf{1}}\end{array}$ & $\begin{array}{c}\text { Diagonal } \\
\text { Anharmonicity } \\
\Delta_{\boldsymbol{i}} / \mathbf{c m}^{-\mathbf{1}}\end{array}$ & $\begin{array}{c}\text { FFCF } \\
\Delta_{\mathbf{1}} / \mathbf{c m}^{-1} \\
\tau_{1}=0.3 \mathrm{ps}\end{array}$ & $\begin{array}{c}\text { FFCF } \\
\mathbf{\Delta}_{\mathbf{2}} / \mathbf{c m}^{-1} \\
\tau_{2}=50 \mathrm{ps}\end{array}$ & $\begin{array}{c}\text { Diagonal } \\
\mathbf{T}_{\mathbf{1}} / \mathbf{p s}\end{array}$ & $\begin{array}{c}\text { Kinetic } \\
\text { Model }\end{array}$ \\
\hline P2 & 1090 & 7 & 11 & 3 & 1.1 & $\tau_{\mathrm{d}}=2.0 \mathrm{ps}$ \\
\hline L1 & 1078 & 9 & 9 & 8 & 0.6 & $\mathrm{k}_{\mathrm{d}}=0.5 \mathrm{ps}^{-1}$ \\
\hline L2 & 1051 & 9 & 7 & 4 & 1.1 & $\tau_{\mathrm{b}}=10 \mathrm{ps}$ \\
\hline R1 & 1018 & 10 & 8 & 10 & 1.1 & $\mathrm{k}_{\mathrm{b}}=0.1 \mathrm{ps}$ \\
\hline R2 & 979 & 12 & 7 & 6 & 1.2 & Coupling \\
\hline L3 & 970 & 12 & 7 & 6 & 1.0 & $\Delta_{i j}=12 \mathrm{~cm}^{-1}$ \\
\hline
\end{tabular}

Table S2: Parameters of the backbone modes for salmon DNA in a CTMA film at $92 \%$ r.h.

\begin{tabular}{|c|c|c|c|c|c|c|}
\hline Mode & $\begin{array}{c}\text { Frequency } \\
\boldsymbol{v} / \mathbf{c m}^{-1}\end{array}$ & $\begin{array}{c}\text { Diagonal } \\
\text { Anharmonicity } \\
\Delta_{i} / \mathbf{c m}^{-1}\end{array}$ & $\begin{array}{c}\text { FFCF } \\
\mathbf{\Delta}_{\mathbf{1}} / \mathbf{c m}^{-\mathbf{1}} \\
\tau_{1}=0.3 \mathrm{ps}\end{array}$ & $\begin{array}{c}\text { FFCF } \\
\mathbf{\Delta}_{\mathbf{2}} / \mathbf{c m}^{-\mathbf{1}} \\
\tau_{2}=50 \mathrm{ps}\end{array}$ & $\begin{array}{c}\text { Diagonal } \\
\mathbf{T}_{\mathbf{1}} / \mathbf{p s}\end{array}$ & $\begin{array}{c}\text { Kinetic } \\
\text { Model }\end{array}$ \\
\hline P2 & 1089 & 11 & 9 & 7 & 0.7 & $\tau_{\mathrm{d}}=2.5 \mathrm{ps}$ \\
\hline L1 & 1068 & 11 & 7 & 6 & 0.8 & $\mathrm{k}_{\mathrm{d}}=0.4 \mathrm{ps}^{-1}$ \\
\hline L2 & 1052 & 11 & 7 & 7 & 0.9 & $\tau_{\mathrm{b}}=10 \mathrm{ps}$ \\
\hline R1 & 1018 & 8 & 7 & 12 & 1.0 & $\mathrm{k}_{\mathrm{b}}=0.1 \mathrm{ps}$ \\
\hline R2 & 975 & 9 & 6 & 6 & 1.0 & Coupling $^{-1.0}$ \\
\hline L3 & 964 & 8 & 7 & 6 & 1.1 & $\Delta_{i j}=9 \mathrm{~cm}^{-1}$ \\
\hline
\end{tabular}

Table S3: Parameters of the backbone modes for salmon DNA in a CTMA film at 0\% r.h.

\begin{tabular}{|c|c|c|c|c|c|l|}
\hline Mode & $\begin{array}{c}\text { Frequency } \\
\mathbf{v} / \mathbf{c m}^{-1}\end{array}$ & $\begin{array}{c}\text { Diagonal } \\
\text { Anharmonicity } \\
\Delta_{\boldsymbol{i}} / \mathbf{c m}^{-\mathbf{- 1}}\end{array}$ & $\begin{array}{c}\text { FFCF } \\
\Delta_{\mathbf{1}} / \mathbf{c m}^{-\mathbf{1}} \\
\tau_{1}=0.3 \mathrm{ps}\end{array}$ & $\begin{array}{c}\text { FFCF } \\
\mathbf{\Delta}_{\mathbf{2}} / \mathbf{c m}^{-1} \\
\tau_{2}=50 \mathrm{ps}\end{array}$ & $\begin{array}{c}\text { Diagonal } \\
\mathbf{T}_{\mathbf{1}} / \mathbf{p s}\end{array}$ & $\begin{array}{c}\text { Kinetic } \\
\text { Model }\end{array}$ \\
\hline P2 & 1089 & 12 & 5 & 13 & 0.8 & $\tau_{\mathrm{d}}=3.3 \mathrm{ps}$ \\
\hline L1 & 1065 & 8 & 3 & 8 & 0.9 & $\mathrm{k}_{\mathrm{d}}=0.3 \mathrm{ps}^{-1}$ \\
\hline L2 & 1046 & 15 & 4 & 15 & 1.3 & $\tau_{\mathrm{b}}=10 \mathrm{ps}$ \\
\hline R1 & 1010 & 8 & 4 & 9 & 1.1 & $\mathrm{k}_{\mathrm{b}}=0.1 \mathrm{ps}$ \\
\hline R2 & 972 & 9 & 5 & 8 & 1.1 & Coupling $^{-1}$ \\
\hline L3 & 957 & 9 & 6 & 8 & 1.1 & $\Delta_{i j}=7 \mathrm{~cm}^{-1}$ \\
\hline
\end{tabular}




\section{Theoretical model of electric interactions at the phosphate-water interface}

The dimethyl phosphate anion $\left(\mathrm{CH}_{3} \mathrm{O}\right)_{2} \mathrm{PO}_{2}{ }^{-}$(DMP) serves as a model system for calculating the electric field at the phosphate-water interface. Due to molecular size and limited conformational space, DMP is accessible by theoretical methods in a rigorous way. DMP constitutes the smallest realistic model systems of the phosphodiester moiety in the backbone of nucleic acids and is a commonly employed model to simulate phosphate group properties of biomolecules like DNA or phospholipids. ${ }^{8-13}$ The gradual sophistication of applied methods to DMP, ranging from classical molecular dynamics to ab-initio density functional theory, has advanced the understanding of the molecular factors and intermolecular solute-solvent interactions determining the properties of the phosphate group. In particular the observed correlation of backbone conformation and phosphodiester stretching modes in the vibrational spectra of DNA (A vs. B form) and RNA has been explained on the basis of calculated frequencies for the DMP fragment, ${ }^{9}$ demonstrating the applicability of DMP as model of the phosphodiester backbone of DNA.

Intermolecular interaction potentials are simulated with the effective fragment potential (EFP) mode $^{14}$ where electrostatic contributions define the electric fields at the phosphate water interface. Within the EFP model the electrostatic potential due to static multipoles is expanded at atom centers and bond mid-points up to octupole moments, polarization due to induction is treated self-consistently in the field of other fragments via induced dipoles distributed on the centroids of localized molecular orbitals. To avoid polarization collapse a Gaussian damping as implemented in libefp is employed. The electric field with contributions from static multipoles and induced dipoles is evaluated up to quadrupole moments. Configurations were generated by a MD trajectory (TIP5P water model ${ }^{15}$, one $\mathrm{K}^{+}$counter ion, Charmm 27 force field ${ }^{16,17}$, DMP partial charges from Ref. 8). Equilibration was performed as described elsewhere, ${ }^{18}$ the first 100 ps of the production run were further employed for equilibration. The last $900 \mathrm{ps}$ of the $1 \mathrm{~ns}$ trajectory were used for EFP field evaluation $(\Delta \mathrm{t}=10$ fs) for a droplet of DMP surrounded by water molecules having at least one atom within a sphere of radius $\mathrm{R}=1.6 \mathrm{~nm}(\sim 250$ water molecules $)$. Induced dipoles are converged within the entire DMP-water droplet followed by EFP field evaluation for a given R. For consistency with the rigid-body model EFP, during MD the DMP conformation was restricted to the $g g$ conformer as prevalent species in $\mathrm{DNA}^{9}$ with the SHAKE algorithm. EFP2 parameters of DMP were generated with the MAKEFP routine as implemented in the GAMESS program package. ${ }^{19}$

Fig. S7 presents the electric field $\mathrm{E}_{\mathrm{C} 2}$ (absolute value) experienced by the $\mathrm{PO}_{2}$ group as projection on the $\mathrm{C} 2$ axis ( $\mathrm{O} \ldots \mathrm{O}$ midpoint) as a function of solvent shell radius $\mathrm{R}$ (top). We compare the radial screening of $\mathrm{E}_{\mathrm{C} 2}$ to the reported radial relative permittivity of Young et al. for the DNA phosphate-water interface (bottom). ${ }^{20}$ We find that the total electric field $\mathrm{E}_{\mathrm{C} 2}$ experienced by the $\mathrm{PO}_{2}$ is rather high $(-92.7 \mathrm{MV} / \mathrm{cm}$, blue line) with the main contribution (76.7\%) arising from static multipoles (El., green line) and noticeable polarization contributions due to induced dipoles (red line). The fluctuation amplitude of the interfacial electric field is characterized by a Gaussian distribution with $\sigma=\Delta \mathrm{E}_{\text {theory }} \approx 24.8 \mathrm{MV} / \mathrm{cm}$. The first water solvation shell of DMP (first minimum of P...O RDF: $0.4 \mathrm{~nm}, 8.9$ molecules on average, $\mathrm{PO}_{2}$ oxygen populated by 3.2 water) is responsible for the main part of $\mathrm{E}_{\mathrm{C} 2}$ 
experienced by the $\mathrm{PO}_{2}$ group $(-70.0 \mathrm{MV} / \mathrm{cm})$. Accordingly $\sim 6$ water molecules generate the main part of $\mathrm{E}_{\mathrm{C} 2}$ in agreement with the findings in Ref. 21. The second shell of water molecules $(0.45-0.7 \mathrm{~nm})$ is responsible for the large remainder of field strength while contributions of water molecules further apart are minor $(6 \%)$.

Our results compare reasonably well with the sigmoidal shape of the radial relative dielectric permittivity at the DNA-water interface reported in Ref. 20. The largest deviations occur for small distances $(\mathrm{R}<0.35 \mathrm{~nm})$ where the dielectric permittivity evolves more smoothly. In contrast, the radial relative dielectric permittivity with respect to $\mathrm{PO}_{2}$ groups of the DNA backbone (BB., blue line in Fig. S7 - bottom) converges at distances of $\mathrm{R}=0.75-0.8 \mathrm{~nm}$, in agreement with our findings of the radial electric field screening. The total relative dielectric permittivity around DNA is somewhat modified due to the major and minor groove, presumably due to water immobilization (Tot., green line Fig. S7 bottom) which is not accounted for by the DMP model. In summary, Fig. S7 allows a comparison of the simulated electric field experienced by the $\mathrm{PO}_{2}$ group of DPM in aqueous solution with available data on the DNA.
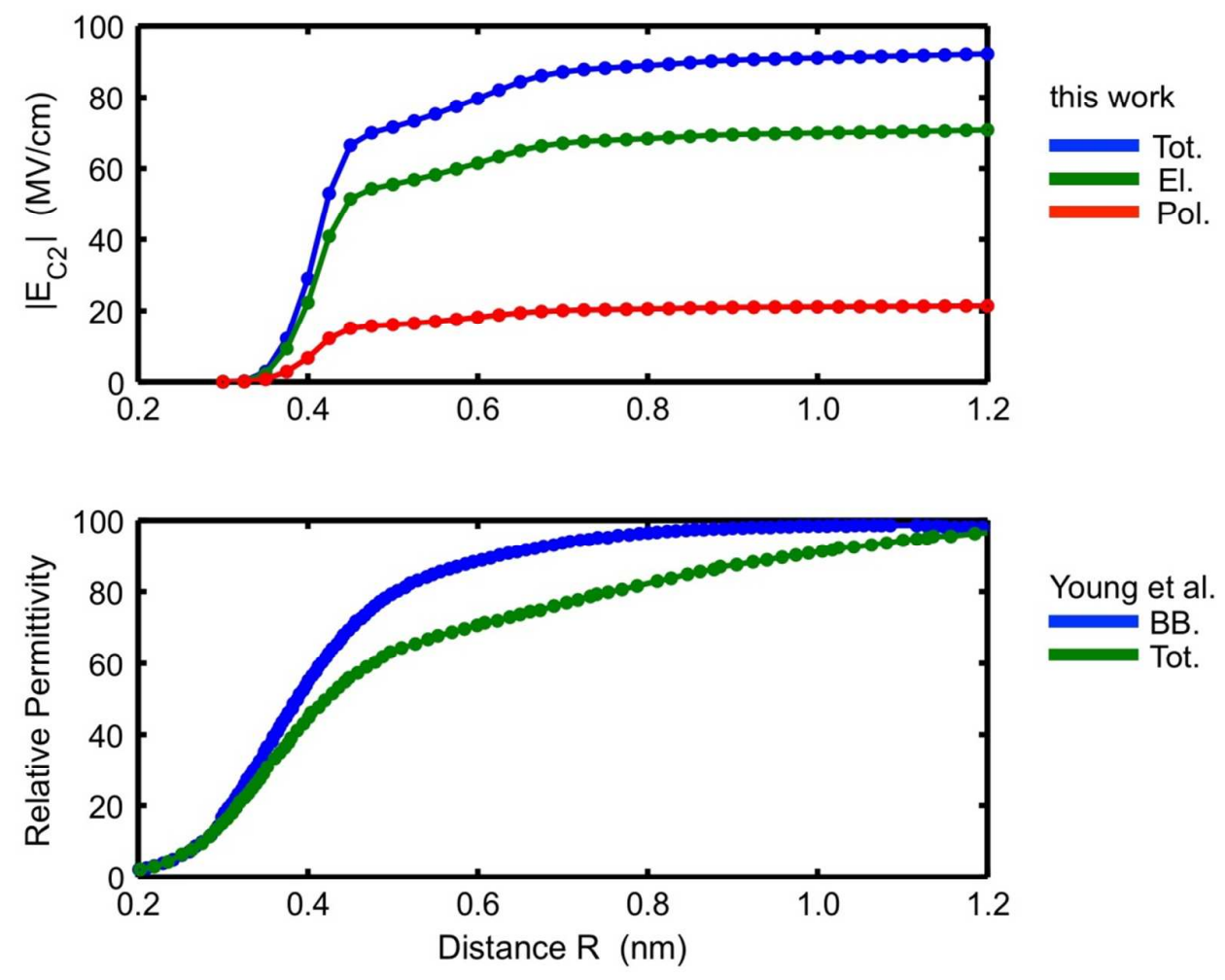

Figure S7. Radial dependence of electric fields. Upper panel: Electric field $\mathrm{E}_{\mathrm{C} 2}$ projected on the $\mathrm{C} 2$ axis of the $\mathrm{PO}_{2}$ group in hydrated DMP. The absolute field strength averaged over 0.9 $\mathrm{ns}$ is plotted as a function of the radial distance $\mathrm{R}$. The blue curve gives the total field, consisting of the electrostatic (green curve) and the polarization contribution from induced dipoles (red curve). Lower panel: Relative dielectric permittivity around DNA from Ref. 20 (blue curve: backbone (BB), green curve: total). 


\section{References}

(1) Tanaka, K.; Okahata, Y. A DNA-Lipid Complex in Organic Media and Formation of an Aligned Cast Film. J. Am. Chem. Soc. 1996, 118, 10679-10683.

(2) Dwyer, J. R.; Szyc, Ł.; Nibbering, E. T. J.; Elsaesser, T. Note: An Environmental Cell for Transient Spectroscopy on Solid Samples in Controlled Atmospheres. Rev. Sci. Instrum. 2013, 84, 036101/1-2.

(3) Hamm, P.; Zanni, M. Concepts and Methods of 2D Infrared Spectroscopy; Cambridge University Press: Cambridge, 2011.

(4) Cowan, M. L.; B. D. Bruner, B. D.; Huse, N.; Dwyer, J. R.; Chugh, B.; Nibbering, E. T. J.; Elsaesser, T.; Miller, R. J. D. Ultrafast Memory Loss and Energy Redistribution in the Hydrogen Bond Network of Liquid $\mathrm{H}_{2} \mathrm{O}$. Nature 2005, 434, 199-202.

(5) Asplund, M. C.; Zanni, M. T.; Hochstrasser, R. M. Two-Dimensional Infrared Spectroscopy of Peptides by Phase-Controlled Femtosecond Vibrational Photon Echoes. Proc. Natl. Acad. Sci. U.S.A. 2000, 97, 8219-8224.

(6) Kaindl, R. A.; Wurm, M.; Reimann, K.; Hamm, P.; Weiner, A. M.; Woerner, M. Generation, Shaping, and Characterization of Intense Femtosecond Pulses Tunable from 3 to $20 \mu \mathrm{m}$. J. Opt. Soc. Am. B 2000, 17, 2086-94.

(7) Siebert, T.; Guchhait, B.; Liu, Y.; Costard, R.; Elsaesser, T. Anharmonic Backbone Vibrations in Ultrafast Processes at the DNA-Water Interface. J. Phys. Chem. B 2015, 119, 9670-9677.

(8) Florián, J.; Baumruk, V.; Štrajbl, M.; Bednárová, L.; Štépánek, J. IR and Raman Spectra, Conformational Flexibility, and Scaled Quantum Mechanical Force Fields of Sodium Dimethyl Phosphate and Dimethyl Phosphate Anion. J. Phys. Chem. 1996, 100, 1559-1568.

(9) Guan, Y.; Thomas Jr., G. J. Vibrational Analysis of Nucleic Acids. III. ConformationDependent Raman Markers of the Phosphodiester Backbone Modeled by Dimethyl Phosphate. J. Mol. Struct. 1996, 379, 31-41.

(10) Florián, J.; Štrajbl, J. M.; Warshel, A. Conformational Flexibility of Phosphate, Phosphonate, and Phosphorothioate Methyl Esters in Aqueous Solution. J. Am. Chem. Soc. 1998, 120, 7959-7966.

(11) Kuo, I. F.; Tobias, D. J. Electronic Polarization and Hydration of the Dimethyl Phosphate Anion: an Ab-Initio Molecular Dynamics Study. J. Phys. Chem. B 2001, 105, 5827-5832.

(12) Ibargüen, C.; Manrique-Moreno, M.; Hadad, C. Z.; David, J.; Restrepo. A. Microsolvation of Dimethylphosphate: a Molecular Model for the Interaction of Cell Membranes with Water. Phys. Chem. Chem. Phys. 2013, 15, 3203-3211.

(13) Andrushchenko, V.; Benda, L.; Páv, O.; Dračínský, M.; Bouř, P. Vibrational Properties of the Phosphate Group Investigated by Molecular Dynamics and Density Functional Theory. J. Phys. Chem. B 2015, 119, 10682-10692.

(14) Kaliman, I. A.; Slipchenko, L. V. LIBEFP: A New Parallel Implementation of the Effective Fragment Potential Method as a Portable Software Library. J. Comp. Chem. 2013, 34, 2284-2292. 
(15) Mahoney, M. W.; Jorgensen, W. L. A Five-Site Model for Liquid Water and the Reproduction of the Density Anomaly by Rigid, Nonpolarizable Potential Functions. $J$. Chem. Phys. 2000, 112, 8910-8922.

(16) Hess, B.; Kutzner, C.; van der Spoel, D.; Lindahl, E. GROMACS 4: Algorithms for Highly Efficient, Load-Balanced, and Scalable Molecular Simulation. J. Chem. Theory Comput. 2008, 4, 435-447.

(17) MacKerell Jr., A. D.; Banavali, N. M.; Foloppe, N. Development and Current Status of the CHARMM Force Field for Nucleic Acids. Biopolymers 2000, 56, 257-265.

(18) Costard, R.; Tyborski, T.; Fingerhut, B. P.; Elsaesser, T. Ultrafast Phosphate Hydration Dynamics in Bulk $\mathrm{H}_{2}$ O. J. Chem. Phys. 2015, 142, 212406/1-9.

(19) Gordon, M. S.; Schmidt, M. W. Advances in Electronic Structure Theory: GAMESS a Decade Later. In Theory and Applications of Computational Chemistry: the First Forty Years; Dykstra, G. E., Frenking, G., Kim, K. S., Scuseria. G. E., Eds.; Elsevier: Amsterdam, 2005, pp. 1167-1189.

(20) Young, M. A.; Jayaram, B.; Beveridge, D. L. Local Dielectric Environment of B-DNA in Solution: Results from a 14 ns Molecular Dynamics Trajectory. J. Phys. Chem. B 1998, 102, 7666-7669.

(21) Floisand, D. J.; Corcelli, S. A. Computational Study of Phosphate Vibrations as Reporters of DNA Hydration. J. Phys. Chem. Lett. 2015, 6, 4012-4017. 